\title{
Toward Understanding the Effects of Visual- and Force-Feedback on Robotic Hand Grasping Performance for Space Teleoperation
}

\author{
Neal Y. Lii, Zhaopeng Chen, Benedikt Pleintinger, Christoph H. Borst, Gerd Hirzinger, and \\ Andre Schiele
}

\begin{abstract}
This paper introduces a study aimed to help quantify the benefits of limited-performance force-feedback user input devices for space telemanipulation with a dexterous robotic arm. A teleoperated robotic hand has been developed for the European Space Agency by the German Aerospace Center (DLR) for a lunar rover prototype. Studies carried out on this telerobotic system investigated several criteria critical to telemanipulation in space: 1) grasping task completion time, 2) grasping task difficulty, 3) grasp quality, and 4) difficulty level for the operator to assess the grasp quality. Several test subjects were allocated to remotely grasp regular and irregular shaped objects, under different combinations of visual- and forcefeedback conditions. This work categorized the benefits of visual- and force-feedback in teleoperated grasping through several performance metrics. Furthermore, it has been shown that, with local joint-level impedance control, good grasping performance with rigid hard objects can be achieved, even with limited force-feedback information and low communication bandwidth. On the other hand, a performance ceiling was also found when grasping deformable objects, where the limited force-feedback setup cannot sufficiently reflect the object boundary to the teleoperator.
\end{abstract}

\section{INTRODUCTION}

$\mathrm{T}_{\mathrm{s}}$ HERE have been considerable interest in telerobotics system performance evaluation in master-slave teleoperation, due to its wide array of relevant applications. Several studies investigated the added value of inclusion of force reflection and its effects under different visual conditions to operator task performance. Such studies have been conducted with both real physical [1] [2] [3] and simulated virtual [4] [5] slave systems.

Despite the large number of investigations, however, a clear overall consensus or guideline for teleoperation system design remain unavailable, namely: 1) the influence of forcefeedback quality on task performance, 2) the roles of force and vision in the form of combined feedback (e.g. feedback dominance-task relationship), and 3 ) the effects of limited force-feedback information on task performance.

Manuscript received March 9, 2010. This work was supported in part by the European Space Agency, 2200 AG Noordwijk, The Netherlands.

Neal Y. Lii, Zhaopeng Chen, Benedikt Pleintinger, and Christoph H. Borst are with the German Aerospace Center (DLR), Oberpfaffenhofen, 82234 Weßling, Germany (phone: +49-(0)8153-281624; fax: 49-(0)8153281134; e-mail: neal.lii@ dlr.de).

Andre Schiele is with the European Space Agency, 2200 AG Noordwijk, The Netherlands (phone: +31(0)71-565-3760; fax: +31(0)71 565 5419; email: andre.schiele@esa.int).
The effects of limited force-feedback on teleoperation performance are the focus of this presented work. It has been observed in previous work force-feedback can indeed be of benefit to teleoperated tasks [2]. However, a counterpoint has also been raised that limiting force-feedback information may in fact lower operator task performance [1].

Understanding the level of refinement in force-feedback information is especially relevant to space teleoperation. One reason is that a space master-slave system is likely to have limited capabilities in bandwidth allocation and transparency, due to overall system resource constraints and possible significant data transmission delay. System cost and physical size, both important design criteria for space applications, could also further reduce the available forcefeedback information for teleoperation. As a result, these systems would be limited to employing limited performance feedback to help perform telemanipulation tasks. Therefore, it is critical to quantify the types and extent of benefit that can be extracted from a low-performance system.

A general purpose space robotic system named Eurobot is under development for exploration purposes at the European Space Agency (ESA) [6]. As a part of defining suitable teleoperation interfaces, it has been deemed necessary to investigate the added value of force reflection under realistic system conditions. The system would employ a dexterous robotic hand and controlled via a data-glove mated to a force-reflection hand exoskeleton.
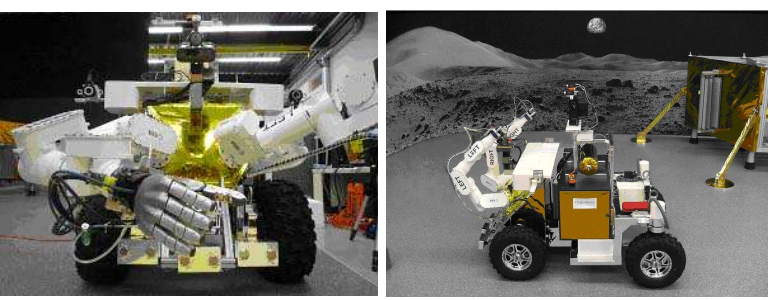

Fig. 1. FFH implementation on the Eurobot Ground Prototype (EGP)

To help assess the functionality of this teleoperated hand system for dexterous grasping tasks, this research aims to establish a concise evaluation metric to clarify the performance gains that can be achieved, under realistic conditions. This paper introduces a study carried out to determine the effects of force- combined with visualfeedback on a realistic range of operator performance metrics - as anticipated for real space teleoperation tasks. As telemanipulation tasks performed in space usually have a combination of various degrees of visual- and forcefeedback, this work would examine several aspects of the effects of different feedback conditions through several 
performance metrics. Besides a larger range of the performance metrics for grasp performance evaluation, this work particularly distinguishes itself from similar works [2] [3], through its emphasis on the use of a small number of force feedback channels compared to the D.O.F. of the end effector (robotic hand), as well as a low-bandwidth communication channel between master and slave system. Finally, this work hopes to serve as an entry into a series of studies to systematically examine the effects of various levels of force-feedback on space telemanipulation tasks.

\section{OVERALl SYSTEM DESCRIPTION}

The proposed system is a further development based on the concepts for teleoperated robotic hand introduced at the German Aerospace Center (DLR), Oberpfaffenhofen, Germany [7]. Its key components consist of a DLR/HIT II five-finger dexterous robotic hand (FFH) [8] [9] and a haptic operator interface from CyberGlove System, as shown in Fig. 2. This system is designed for dexterous object manipulation to be employed on the Eurobot Ground Prototype (EGP), and operated eventually from the space station remotely. The EGP, shown in Fig. 1, is a planetary exploration version including a mobility and navigation subsystem, of the Eurobot [6].

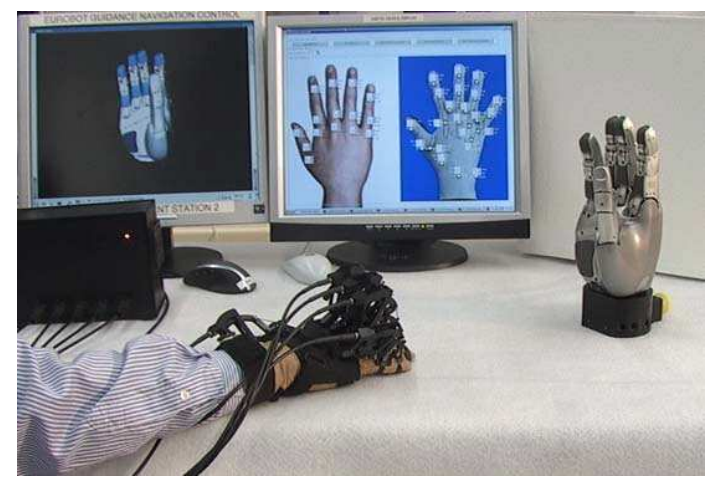

Fig. 2. Experimental environment

\section{A. Master System: User Telemanipulation Input}

The CyberGrasp [10] consists of a data glove for hand gesture measurement, and an exoskeleton for force-feedback. The CyberGrasp hardware can be seen worn by an operator as a part of the experimental environment in Fig. 2.

The data glove utilizes 22 bend sensors with 8-bit resolution to measure the movements of each joint in the hand. As each operator's hand size and form can differ significantly, the system provides the possibility to calibrate the glove sensors to custom fit to each user. Although both the CyberGlove and the FFH attempt to mimic fully the human hand, both are unable to fully duplicate all joints in the human hand. For example, the CyberGlove provides only four sensors to measure five finger abductions, whereas the FFH does not have a roll joint for the opposing thumb. Therefore, the glove sensor data measurement must be appropriately mapped to the robotic hand for improved telemanipulation performance.
The force-feedback exoskeleton composes of five singledirection cable actuators to exert force on each finger to generate opposing force to finger flexion. The forcefeedback exoskeleton's five channels of force in the pullback direction (one for each finger) are significantly fewer than the 22 D.O.F. for the human hand, as well as the 15 D.O.F of the FFH. Furthermore, with a bandwidth of $\sim 90$ $\mathrm{Hz}$, a limitation is also placed on the data transmission rate. These conditions place a significant limit on the forcefeedback information available to the remote operator.

As the dexterous robotic hand is able to provide three torque measurements for each finger, or 15 in total, an appropriate mapping of forces to be reflected from the robotic hand to the exoskeleton would also be necessary.

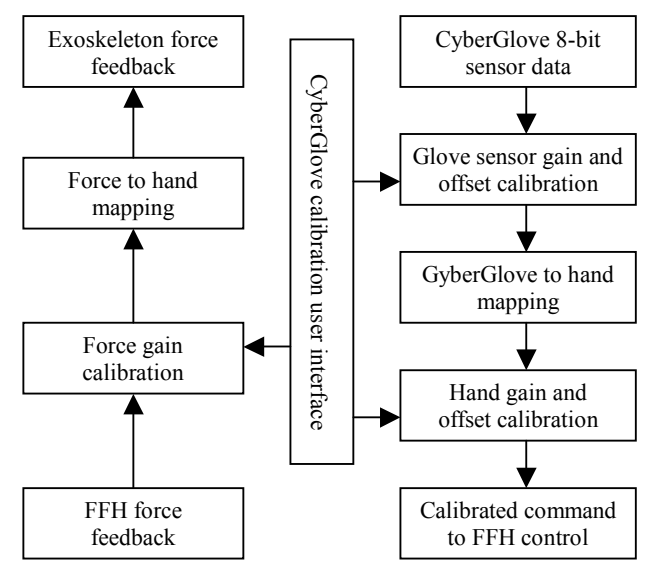

Fig. 3. Flow-chart of software modules for calibration and mapping between data-glove, robotic hand and hand exoskeleton

The glove command and force-feedback mapping algorithms have been modeled in Matlab/Simulink, and operate in the QNX real-time environment via external mode. To help improve operator command and feedback performance, a GUI has been included in the system, in addition to sensor calibration, to adjust the mapped hand response, as well as feedback force gain, as shown on the right hand screen in Fig. 2. Finally, a diagram is shown in Fig. 3 to help clarify the user manipulation and forcefeedback system design.

\section{B. Slave System - Robotic Hand}

The DLR-HIT II five-finger dexterous robotic hand (FFH) was jointly developed by DLR, and the Harbin Institute of Technology, Harbin, China [8] [9]. It is based on the technology and concepts developed on the DLR Hand II [11], and maximized the use of off-the-shelf components to help explore the feasibility of series production.

The FFH consists of five identical modular fingers, serving as four fingers and one opposing thumb. Each finger has three DOF, with coupled intermediate and distal joints, and decoupled proximal and abduction actuation with a maximum speed of $180 \mathrm{deg} / \mathrm{s}$. Each finger is capable of a maximum tip force of $9 \mathrm{~N}$. The FFH can be seen as a part of the system setup in Fig 7.

The FFH can be implemented with Cartesian- or joint-space impedance controllers [9] for hand grasping tasks. For the 
application of glove-controlled object telemanipulation on the Eurobot, human finger joints would be mapped onto the robotic hand to simulate the grasp gestures commanded by the operator's hand. In this case, the joint-space controller would be the more able to closely simulate the intention of the human operator. The implementation of the proposed controller is explained in more detail as follows:

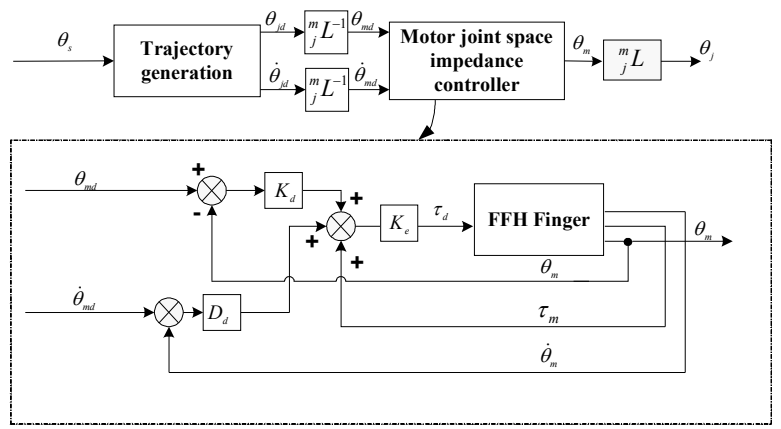

Fig4. The robotic hand joint space model. Where:

$\theta_{s}$ is the operator desired joint angle,

$\theta_{j d}$ is the desired joint angle post trajectory generation,

$\dot{\theta}_{j d}$ is the desired joint angular rate post trajectory generation,

$\theta_{m d}$ is the desired motor angle,

$\dot{\theta}_{m d}$ is the desired motor angular rate,

$K_{d}$ is the desired stiffness,

$D_{d}$ is the desired damping,

$\tau_{m}$ is the actual motor torque,

$\theta_{m}$ is the actual motor angle,

$\dot{\theta}_{m}$ is the actual motor angular rate,

$\theta_{j}$ is the actual FFH finger joint angle,

$K_{e}$ is the impedance behavior between the finger joint and external torque,

and $L$ represents the motor-joint forward kinematics.

The robot hand dynamic model is as expressed below [12]:

$$
\begin{aligned}
& M(q) \ddot{q}+C(q, \dot{q}) \dot{q}+g(q)=\tau+\tau_{\text {ext }} \\
& B \ddot{\theta}+\tau+\tau_{f}=\tau_{m} \\
& \tau=K(\theta-q)
\end{aligned}
$$

$M(q), C(q, \dot{q}) \dot{q}$, and $g(q)$ represent the inertia matrices, centrifugal term, and gravity term, respectively. The joint torque vector is given by $K(\theta-q)$, where $\theta$ indicates the vector of the motor angle divided by the gear ratio, and $q$ represents the link side joint angle. $K$ and $B$ are diagonal matrices which contain the joint stiffness, and the motor inertia multiplied by the gear ratio squared. $\tau$ and $\tau_{f}$ are external torque vector and friction torque vector, respectively. The generalized actuator torque vector, $\tau_{m}$, is considered as the control input.

The goal of the impedance controller is to achieve a desired dynamic behavior with respect to external forces and torques acting on the link side of the finger, given by a desired stiffness parameter $K_{d}$, as well as a desired damping parameter $D_{d}$ [13]. For passivity considerations, in case that the desired impedance behavior is defined in the joint coordinate systems, a motor position based PD-controller can be implemented as the following:

$$
\tau_{m}=-K_{d}\left(\theta-\theta_{s}\right)-D_{d} \dot{\theta}
$$

where $\theta_{s}$ represents a desired configuration in joint space. Together with (2) and (3), the following closed loop equations can be arrived:

$$
\begin{aligned}
& M(q) \ddot{q}+C(q, \dot{q}) \dot{q}+g(q)=\tau+\tau_{\text {ext }} \\
& B \ddot{\theta}+D_{d} \ddot{\theta}+K_{d} \widetilde{\theta}+\tau+\tau_{f}=0
\end{aligned}
$$

where

$$
\widetilde{\theta}=\theta-\theta_{s}
$$

The implementation of the proposed controller is as shown in Fig. 4.

The controller is implemented in Matlab/Simulink, and runs in the QNX real-time environment via external mode. A graphic user interface allows the operator to control the FFH's system settings remotely. A remote viewer of the FFH is also built into the FFH GUI, which provides a streaming display of the robotic hand's current gesture based on the position sensors' measurements. The remote viewer of the FFH is shown in Fig. 2 as a part of the experimental environment on the monitor to the left.

\section{Overall System Architecture}

The system architecture of the CyberGrasp teleoperated FFH consists of two sides: the commander side, and the EGP side, where the FFH is installed. The communication between the sides is provided via a wireless LAN link. The CyberGrasp System CyberGlove and exoskeleton are installed on the command side for user haptic command input and force-feedback. This side of the system also provides the operator with the user interfaces both for the CyberGrasp and the FFH, to allow the operator to make necessary adjustments to the system for different telemanipulation tasks. A QNX real-time mini-rack computer is implemented on the EGP Side for the control of the FFH. The system may be operated in wired or wireless mode, depending on task requirements.

The robotic hand controller operates at a rate of $5 \mathrm{KHz}$. The transmission rate to the CyberGrasp is at above $1 \mathrm{KHz}$ in wired network configuration, and about $500 \mathrm{~Hz}$ in wireless configuration. However the bottleneck of the system refresh rate lies in the CyberGrasp controller, which updates sensor measurements at $90 \mathrm{~Hz}$ intervals.

\section{EXPLORATORY FORCE-FEEDBACK GRASPING EXPERIMENTAL STUDY AND APPROACH}

\section{A. Experiment Subjects}

Three human operator subjects were allocated to perform a series of teleoperated grasping tasks of different objects. Each subject is of different a level of knowledge on telemanipulation and the experimental setup, ranging from 
expert and high familiarity with the system to a novice user. The spread in expertise allowed a more full-spectrum examination of the teleoperator response and performance.

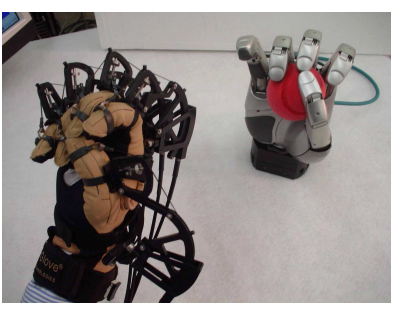

Teleoperated FFH grasping soft ball

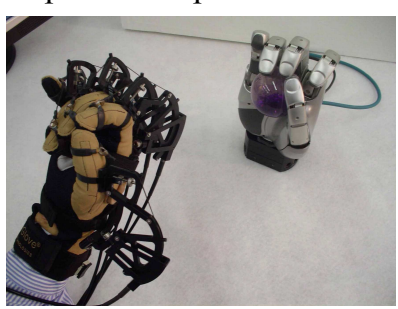
hard ball
Teleoperated FFH grasping

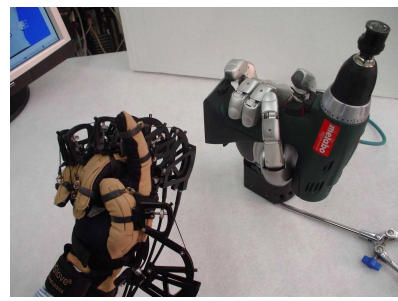

Teleoperated FFH grasping pistol grip tool

Fig. 5. Different test grasp objects being grasped as commanded by the CyberGrasp user command

\section{B. Selection of Objects for Teleoperated Grasping}

Grasping of regular and irregular shaped objects was examined. For this study, the sphere shape was chosen as a representative regular shape. A hard ball was used to simulate a rigid regular shaped object, and a soft ball to simulate a deformable object. The balls are of similar dimensions of about $6.7 \mathrm{~cm}$.

The pistol grip tool was selected to represent an irregular shape object for its relevance in space application, particularly in extra-vehicular operations from the International Space Station (ISS). A power drill was used to simulate a pistol grip tool due to its similarities in form, size, and trigger position to actual pistol grip tools.

Fig. 5 shows the three types of test grasp objects being grasped by the FFH teleoperated through the CyberGrasp haptic user command.

\section{Test Sequence and Performance Metrics}

In order to examine the effects of visual- and forcefeedback on grasping performance, each of the three test subjects were required to telemanipulate grasps of the regular and irregular shaped objects described above, under various combinations of feedback conditions. These included:

- Optimal vision with force-feedback

- Obstructed vision with force-feedback

- Optimal vision without force-feedback

- Obstructed vision without force-feedback

Each grasp object-feedback condition combination was repeated 10 times to help collect sufficient grasp samples. For each grasp operation, the 1) time to completion, 2) grasp quality, and 3) grasp assessment correctness were recorded as objective metrics. Finally, for each grasp object-feedback condition combination, scalar ratings of 1) perceived grasp task difficulty, and 2) grasp quality assessment difficulty are given by each test subject as subjective metrics. The data collected and observations are analyzed both qualitatively and quantitatively, and discussed in the following sections.

\section{RESULTS AND ANALYSIS}

In order to analyze data collected from the telemanipulation grasping experiments, ANalysis Of VAriance (ANOVA) is employed in this section, which allows the definition of threshold for significant variances in sample means. In the following, variation probabilities under the $5 \%$ level are considered significant.

\section{A. Time to Complete Grasping Task}

Possibly due to the relatively low number of test subjects, experimental results have shown no significant difference in task completion time in relation to different objects, as shown in Fig. 6. A weak trend is visible towards more time required for task completion as a result of less feedback information, both visual and force.

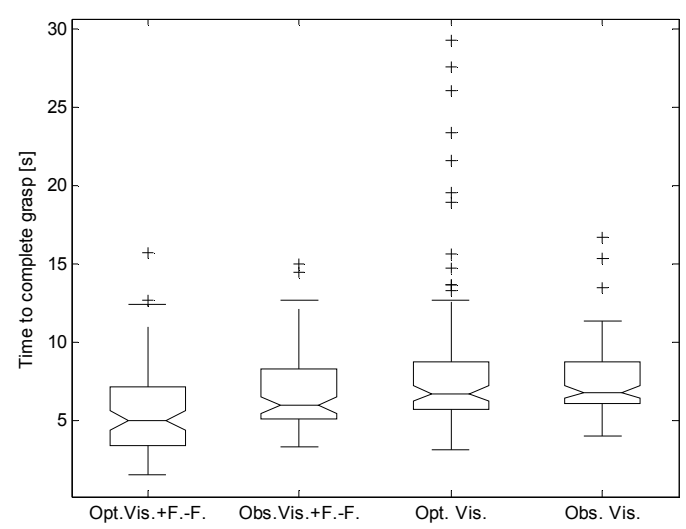

Fig. 6. Box plot of group measurements of time required until grasp completion. The experiment conditions are shown as: Opt.Vis.+F.-F. (optimal vision including force-feedback), Obs.Vis.+F.-F. (obstructed vision including force-feedback), Opt.Vis. (optimal vision alone), Ohs. Vis. (ohstructed vision alone).

\section{B. Perception of Task Difficulty}

Fig. 7 shows a trend of overall increasing difficulty with the compounding loss of feedback information. This is particularly clear when left with only obstructed vision of the grasping task. Clear statistical significance can be seen compared to other conditions of optimal vision with forcefeedback $(\mathrm{p}=0.003)$, obstructed vision with force-feedback $(\mathrm{p}=0.002)$, and optimal vision setup $(\mathrm{p}=0.008)$. 


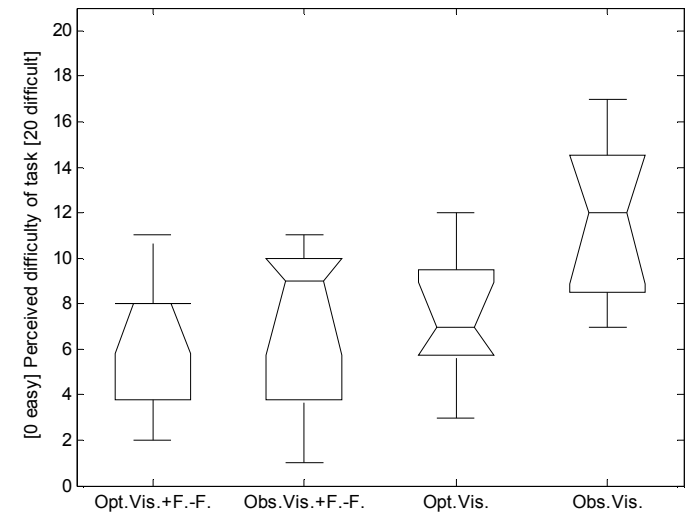

Fig. 7. Box plots of group measurements of perceived task difficulty over the four experiment conditions. The difficulty of task is graded by the subjects on a scale of 0 to 20 , with 0 being the easiest, and 20 the most difficult

When examining vision as the sole feedback criteria, it plays a crucial role in perceived task difficulty. This is clearly demonstrated in Fig. 8, as vision obstruction increases the difficulty of the task significantly $(\mathrm{p}=0.019)$. All test objects also noted their increased reliance on the robotic hand virtual viewer provided when performing grasping tasks under obstructed visual-feedback.

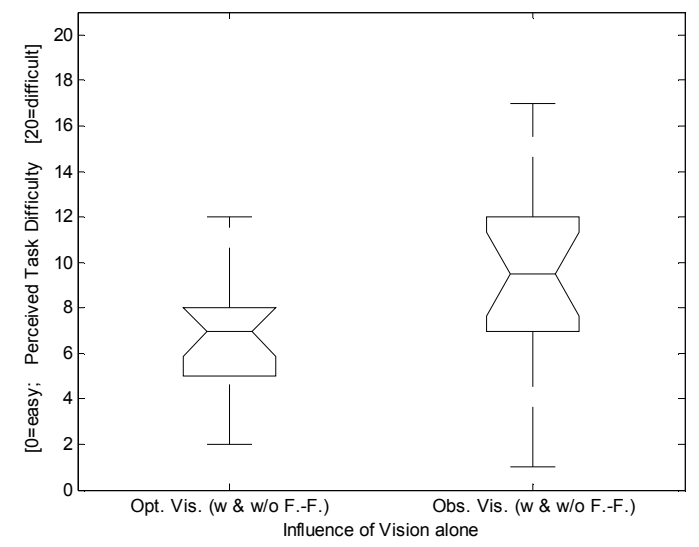

Fig. 8. Influence of vision alone to perceived task difficulty. Obstructed vision significantly increases the perceived difficulty of the task $(\mathrm{p}=0.019)$. Both conditions included tests with and without force-feedback.

Force-feedback also demonstrates a strong influence on the perceived task difficulty. Without force-feedback, the task difficulty increases significantly $(\mathrm{p}=0.026)$, as shown in Fig. 9.

\section{Assessment of Grasp Quality}

The grasp quality assessment reflects the confidence of the operator in his/her knowledge of the state of the grasp. The test subjects were asked to assess whether a grasping task has succeeded or failed upon task completion. The assessment is immediately confirmed by an experiment facilitator by an attempt to remove the grasped object from the FFH.

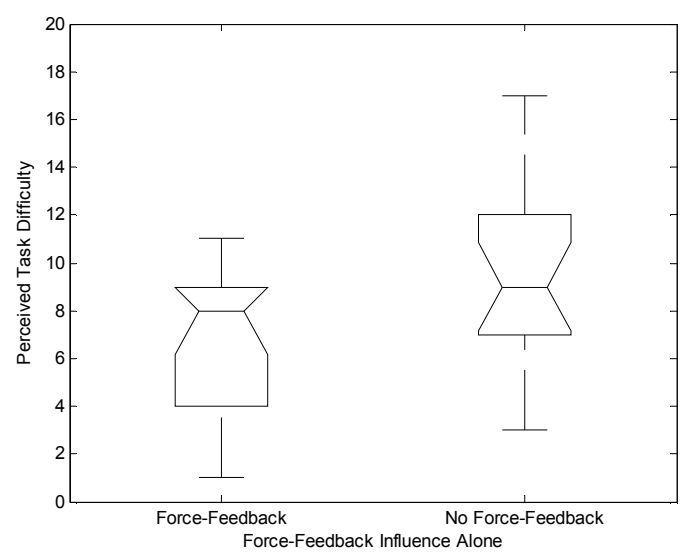

Fig. 9. Influence of force-feedback alone on perceived task difficulty. Without force-feedback, difficulty increases significantly $(\mathrm{p}=0.026)$. Both conditions included optimal and obstructed vision.

\section{1) Difficulty level of grasp quality assessment}

As shown in Fig. 10, the object itself gives clues about grasp performance. It was easier to assess grasp quality with the pistol grip tool, than with the hard or soft balls $(p=0.005)$. This aspect would be further examined in the following subsection.

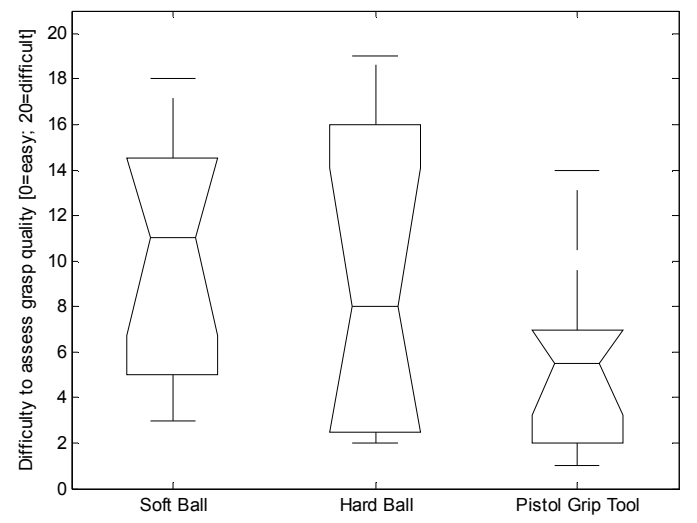

Fig. 10. Group ratings on difficulty to assess grasp quality remotely during operations. Dependance on the object to be grasped.

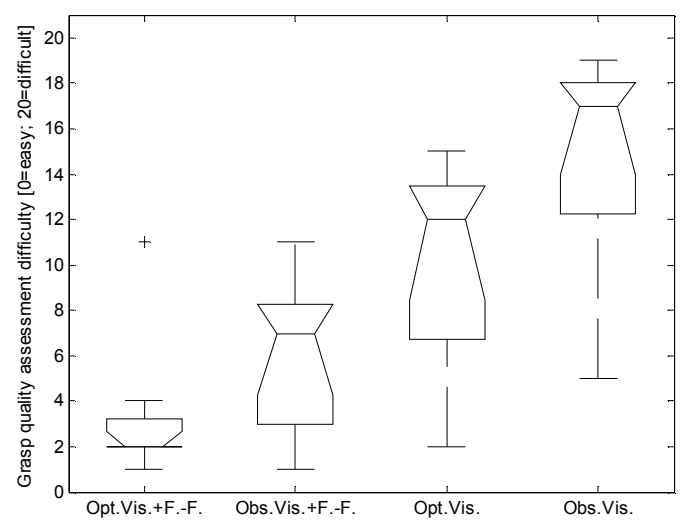

Fig. 11. Group results for the difficulty levels to assess grasp quality under different experimental feedback condition 
Fig. 11 shows that a good vision on the worksite, along with the force-feedback allows the best assessment of the actual grasp situation. With decreasing levels of feedback information toward obstructed vision without forcefeedback, the assessment of the grasp performance becomes increasingly difficult. For example, obstructed vision without force-feedback is significantly more difficult than optimal vision with force-feedback $(\mathrm{p}<0.001)$. A more detailed examination to isolate the effects of visual- and forcefeedback has shown that visual-feedback played a relatively minor role with $\mathrm{p}=0.028$, whereas force-feedback played a significant role in grasp quality assessment with $\mathrm{p}<0.001$.

2) Percentage of correct assessments of grasp success

As shown in Fig. 12, the pistol grip tool demonstrated $100 \%$ correct assessments. This is likely due to the complex and rigid shape, which provides the operator with more clues to arrive at correct judgments. On the other hand, the soft ball was deemed the most difficult to assess. One main reason, as pointed out by the test subjects, was the difficulty to tell if a firm grasp has been achieved as a result of a soft, deformable shape. This shows the limitation of a lowperformance force-feedback system in help the operator to clearly distinguish the object boundary when the object is highly deformable.

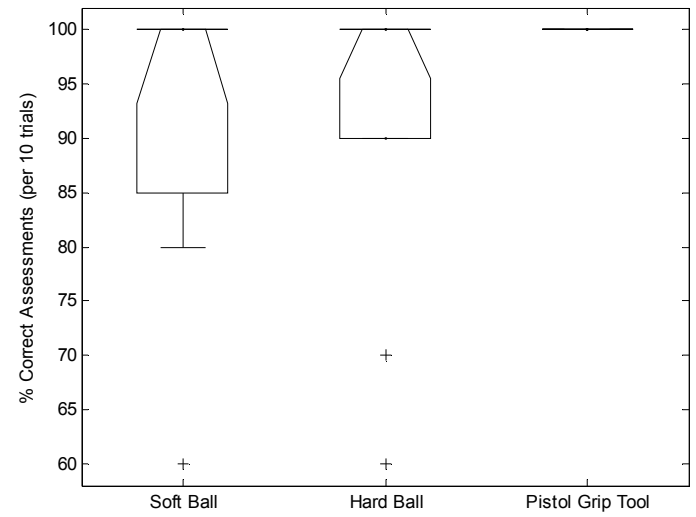

Fig. 12. Group distribution for the condition of obstructed vision with force-feedback. It shows the percent of correctly estimated grasp situations, within each trial consisting of 10 repetitions for soft ball, hard ball and pistol grip tool.

Fig. 13 shows that assessments were most difficult with obstructed vision alone and best with optimal and/or obstructed vision with force-feedback. Upon closer examination, as shown in Fig. 14, it is clear that assessment difficulty is direct a result of the lack of force-feedback within the task $(\mathrm{p}=0.0121)$, whereas No effect directly due to the different levels of visual-feedback.

\section{Grasping tasks success rate}

As shown in Fig. 15, with the aid of force-feedback information, telemanipulators can achieve nearly $100 \%$ success in grasping task for objects of different shapes and rigidity. This compares favorably against data with no forcefeedback is available, where error rates as high as $20 \%$ have been observed.

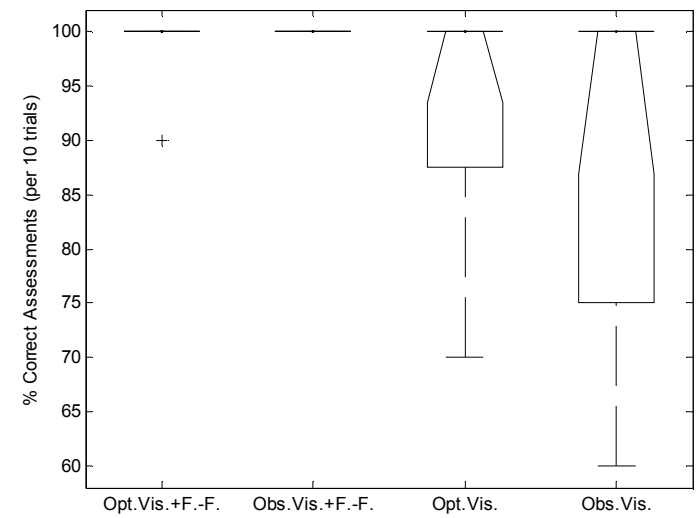

Fig. 13. Group distribution of different feedback conditions. It shows the percentage of correctly estimated grasp situations, consisting of 10 repetitions within each trial.

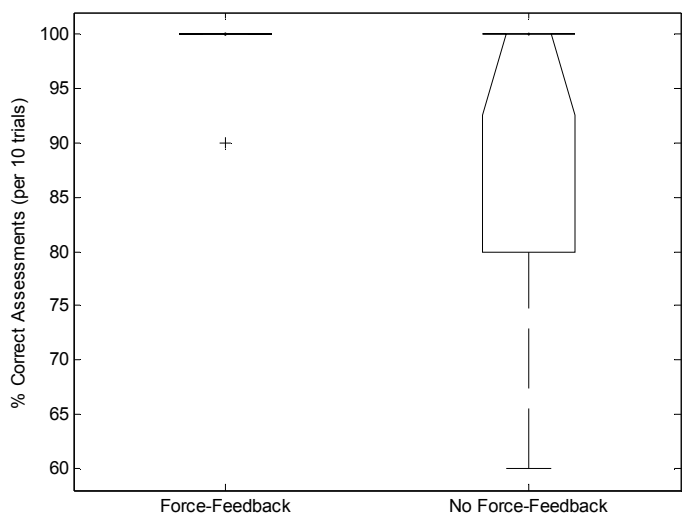

Fig. 14. Group distribution showing the percentage of correct assessments by the test subjects. A comparison is made here between feedback conditions with and without force-feedback.

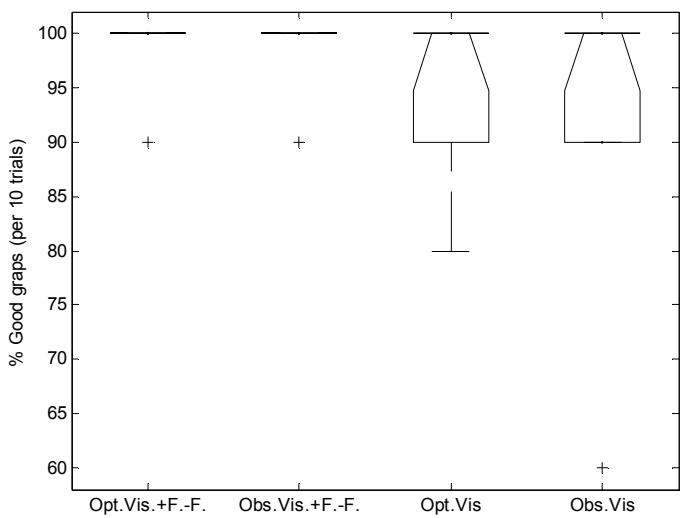

Fig. 15. Percentage of successful grasps within a 10 repetitions trial. Boxplot for the combined results of the entire group is displayed over the four different experiment conditions for all object types.

\section{Discussion AND Final OBSERVATIONS}

Both visual- and force-feedback have shown strong influences on perceived difficulty of the grasping task, with vision playing a slightly more significant role. This is in line 
with previous findings reported, e.g. in [4]. When the vision is obstructed, the inclusion of force-feedback helps significantly reducing task load, as compared to optimal vision without force-feedback.

Force-feedback can also serve the role of a backup feedback source for a fault tolerant redundancy system, necessary for safety-critical applications such as space telerobotics. In this case, the remaining, unimpaired feedback channel changes its role from a cooperative member of a combined feedback to a redundant channel [14]. This also confirms previous findings that forcefeedback makes a bigger contribution to lowering task load with less visual feedback information [2].

The test subjects' increased reliance on the virtual robotic hand viewer in obstructed view conditions points to the need for such features in future space teleoperation implementations. From an investigative point of view, effects of view obstruction, without the aid of a virtual viewer, should be examined, to further distill the effects of force- and visual-feedback.

The form closure that can be achieved with the pistol grip, may have contributed, at least in part, to its associated grasp quality assessment success. This result has also demonstrated the importance of tool form design to help improve ease of tasks performed in space.

This paper has shown that force-feedback plays a critical role in helping the operators assess the quality of the grasp. Fig. 14 gives clear evidence of force-feedback as a key factor in correct grasp success/failure assessment. It was also shown that good vision contributes to this assessment ability. Fig. 11 and Fig. 13 show that a good vision, together with force-feedback produces nearly $100 \%$ correct assessments of the grasp success, whereas for other setups without forcefeedback, this rate can drop down to $60 \%$. It should be specifically mentioned here that this is true for a case in which only $90 \mathrm{~Hz}$ of up-dated signals are produced by the CyberGrasp finger exoskeleton.

The fact that force-feedback contributed less to task performance improvement with the deformable object, has shown the limitation of the low-performance force-feedback device implemented here. While local impedance control helped to achieve stability, the low update rate on the masterside still prohibits the rendering of small changes of contact forces and high dynamic range.

Finally, aside from the data shown here, it should also be mentioned that different strategies of grasping have been observed for subjects of different level of expertise in this experiment. The expert-level subject seemed to focus during each grasp trial on finding a best and optimal grasp. The novice subject in contrast seemed to use the first few grasps to find a best average suitable grasp. Once a good grasp type was deemed discovered, the novice subject repeated the same grasp for the remainder of the same experiment (e.g. grasp of a soft regular shaped object with optimal visual feedback with force-feedback).

\section{CONCLUSION}

This work categorized the benefits of visual- and forcefeedback in teleoperated grasping through a series of grasping performance evaluation metrics. Two findings in this paper have made this work worthwhile to this space teleoperation project. First, through the series of test metrics, it has been clearly shown, that with the aid of local jointlevel impedance control on the end effector, even with limited bandwidth and force-feedback channels, forcefeedback still demonstrated clear benefits in grasping hard objects of regular and complex shapes. The performance benefits have been particularly verified in grasp quality assessment, and reducing grasping task difficulty. Second, a performance border has been identified, where the limitations in bandwidth and force-feedback information would limit grasp performance. This occurs with the grasping of deformable objects, as the operator cannot identify the object boundary, thus making telegrasping more difficult. On the other hand, these findings also suggest that tools for telerobots should be designed to form-fit specifically to the end effector to help improve space teleoperation performance.

\section{FUTURE WORK}

Moving forward, this project would aim to build a more clearly defined and extensive set of test metrics, based on the findings from this study, to further clarify the effects of force-feedback in different operating environments, with more grasp object shapes, a larger pool of test subjects, and ideally also with a range of performances of force-feedback information. Furthermore, as real grasping tasks can only be performed effectively with a robotic arm-hand system. Investigations are underway to get a more holistic view of the effects of visual- and force-feedback in a hand-arm system. The user command would be retrieved by employing an arm exoskeleton system developed for such tasks [15]. Finally, as demonstrated from the experimental results of grasping deformable objects, finer force-feedback information can indeed improve some grasp operations. Therefore, it would be helpful to investigate the possibilities of improving the force-feedback information available through new device developments, while meeting space teleoperation resource constraints.

\section{ACKNOWLEDGMENT}

The Authors would like to express our deepest thanks to Mr. Florian Schmidt of DLR for his tireless support in constructing the proposed system environment. Mr. Peter Meusel of DLR provided valuable logistics assistance throughout the project. Mr. Ralph Bayer provided the mechanical designs for the FFH base adaptor. Dr. Hong Liu of DLR provided useful guidance and suggestions in the application of the FFH. Our sincere gratitude is also goes to Mr. Jan Geerse of Dutch Space, Leiden, The Netherlands, 
for providing the test location and on-site support.

\section{REFERENCES}

[1] B. Hannaford, L. Wood, D. A. McAffee, and H. Zak, "Performance evaluation of a six-axis generalized force-reflecting teleoperator," IEEE Transactions on Systems, Nan, and Cybernetics, Vol. 21, No. 3, May/June 1991, pp. 620-633

[2] M. J. Massimino, and T. B. Sheridan, "Variable force and visual feedback effects on teleoperator man/machine performance," in Proceedings of the NASA Conference on Space Telerobotics, Pasadena, CA, USA, 1989, pp. 89-98

[3] M. L. Turner, R. P. Findley, W. B. Griffin, and M. R. Cutkosky, "Development and testing of a telemanipulation system with arm and hand motion" Proceedings of the ASME International Mechanical Engineering Congress and Exposition, Dynamic Systems and Controls, 69, pp. 1057-1063

[4] O. Gerovichev, P. Marayong, and A. M. Okamura, "The effect of visual and haptic feedback on manual and teleoperated needle insertion," Proceedings of the Fifth International Conference on Medical Image Computing and Computer Assisted Intervention -MICCAI 2002, Lecture Notes in Computer Science (Vol. 2488), T. Dohi and R. Kikinis, Eds., 2002, pp. 147-154

[5] P. Richard, G. Burdea, D. Gomez, P. Coiffet, "A Comparison of haptic, visual and auditive force feedback deformable virtual objects," in Proceedings of the Fourth International Conference on Artificial Reality and Tele-Existence, Tokyo Japan, 1994, pp. 49-62

[6] F. Didot, P. Schoonejans, and R. Stott, "Eurobot Underwater Model Testing the Co-operation Between Human and Robots", The 9th European Space Agency Workshop on Advanced Space Technologies for Robotics and Automation ASTRA 2006, The Netherlands, November 28-30, 2006

[7] C. H. Borst, M. Fischer, S. Haidacher, H. Liu, G. Hirzinger: DLR hand II: experiments and experiences with an anthropomorphic hand. IEEE International Conference on Robotics and Automation, 2003 pp.702-707

[8] H. Liu, K. Wu, P. Meusel, N. Seitz, G. Hirzinger, M. Jin, Y. Liu, S. Fan, T. Lan, and Z. Chen, "Multisensory five-finger dexterous hand: The DLR/HIT Hand II", in IEEE/RSJ International Conference on Intelligent Robots and Systems, 2008, pp. 3692-3697.

[9] Z. Chen, N. Lii, S. Fan, M. Jin, and H. Liu, "Experimental study on impedance control for the five-finger dexterous robot hand DLR-HIT II", manuscript in preparation

[10] Virtual Technologies Inc., Virtual Hand v2.5 Programmer's Guide, 2001

[11] H. Liu, J. Butterfass, M. Grebenstein, "DLR-Hand II: next generation of a dexterous robot hand", in Proceedings of the 2001 IEEE International conference on Robotics I\& Automation, 2001, pp: 109114

[12] R.M. Murray, Z. Li, and S. S. Sastry, A Mathematical Introduction to Robotic Manipulation, CRC Press, 1994

[13] A. Albu-Schaffer, C. Ott, and G. Hirzinger, "A unified passivitybased control framework for position, torque and impedance control of flexible joint robots," The International Journal of Robotics Research, Vol. 26, No. 1, 2007, pp. 5-21

[14] W. Elmenreich, Sensor Fusion in Time-Triggered Systems, PhD Dissertation, Technische Universität Wien, Institut für Technische Informatik, Vienna, Austria, 2002

[15] A. Schiele, "Case Study: The ergonomic EXARM exoskeleton", in Wearable Robots: Biomechatronic Exoskeletons, J.L. Pons, Ed.: John Wiley \& Sons Ltd., 2008, pp. $248-255$ 